Journal of Mathematics and Statistics 6 (3): 265-270, 2010

ISSN 1549-3644

(C) 2010 Science Publications

\title{
Simple Improvement of Momentum Interpolation Equation for Navier-Stoke Equation Solver on Unstructured Grid
}

\author{
Adek Tasri \\ Department of Mechanical Engineering, Andalas University, \\ Kampus Unand Limau Manis, Padang 25163, Indonesia
}

\begin{abstract}
Problem statement: Pressure and velocity decoupling have been source of problem in solving Navier-Stokes and continuity equation particularly in complex collocated grid. The problem of pressure velocity decoupling is usually reduced by using momentum interpolation to calculate mass flux at face of control volume. Equation of momentum interpolation was derived by assumption that the face of cell is equidistant from two neighbor cell centers and face of cell is collinear with two neighbor cell centers. This assumption is not valid in many unstructured grid and cause significant error. Approach: In this article a simple improvement of momentum interpolation for using in unstructured grid was proposed. The improvement was done by added a correction term to original equation. Results: The method was compared with original method in Kovasznay's flow and Laminar Poisseulli Flow. The method was found able to reduce error about $40 \%$ in both cases. Conclusion: The correction added to original momentum interpolation is able to reduce error in Navier-Stokes equation solver on unstructured grid.
\end{abstract}

Key words: Momentum interpolation, unstructured mesh, finite volume

\section{INTRODUCTION}

It is well known that numerical solving of NavierStokes equation in primitive variable on collocated grid produce checkerboard oscillation if central difference is used to approximate face velocity in discretised continuity equation and pressure gradient in momentum equation (Yu and Kawaguchi, 2002). The oscillation is caused by decoupling of pressure and velocity in discretised equation (Miller and Schmidt, 1988). Rhie and Chow (1983) first solved this problem for structured collocated grid by modifying the interpolated face velocities so as to recognize the pressure difference across the face. The momentum interpolation of Rhie and Chow (1983) is popularly used to calculate face velocity in discretised momentum equation for SIMPLE based algorithm since. Later, it was found that the original Rhie and Chow method had some drawback related to dependence of calculation result on under relaxation factor and time step size influence on convergence of the solution as reported by Majumdar (1988) and Choi (1999). The way to solve this problem also been proposed by some author ( $\mathrm{Yu}$ and Kawaguchi, 2002; Nie et al., 2000).

Improvement of Rhie and Chow interpolation in order to improve accuracy was reported by Papageorgakopoulos et al. (2000) and Yu et al. (2002). But both methods are for used in structured grid. So far only very limited number of study related to accuracy of application of the Rhie and Chow (1983) interpolation method on unstructured grid have been published. A popularly used method to implement Rhie and Chow (1983) interpolation method on unstructured grid is based on the way proposed by Mathur and Murthy (1997). Mathur and Murthy (1997) applied momentum interpolation to unstructured grid by assuming the face is laid down inline and in the middle between two neighbor cell centers where the interpolation was claimed formally second order. If the face is not inline and in the middle of two neighbor cell as the case of most unstructured grid the accuracy of interpolation will down to first order. Therefore in this study a simple modification to interpolation of Mathur and Murthy (1997) in order to solve this problem is proposed.

In this study, two examples are used as test case and solve in unstructured grid:

- Kovasznay's flow

- Laminar Poisseulli flow

\section{MATERIAL AND METHODS}

Flow equation: The governing equation of 2D laminar steady flow of incompressible Navier-Stokes fluids in arbitrary domain is continuity and Navier-Stokes equation: 
$\operatorname{div} \rho \overrightarrow{\mathrm{V}}=0$

$\operatorname{div} \rho V_{1} \vec{V}=\operatorname{div}\left(\mu \nabla V_{1}\right)-\frac{\partial P}{\partial x}$

$\operatorname{div} \rho \mathrm{V}_{2} \overrightarrow{\mathrm{V}}=\operatorname{div}\left(\mu \nabla \mathrm{V}_{2}\right)-\frac{\partial \mathrm{p}}{\partial \mathrm{y}}$

Where:

$\mathrm{V}_{1}$ and $\mathrm{V}_{2}=$ Cartesian component of velocity vector $\rho$ and $\mu \quad=$ Density and viscosity respectively

The momentum equation can then be written in form of general transport equation for scalar $\phi$ :

$$
\operatorname{div} \rho \phi \overrightarrow{\mathrm{V}}=\operatorname{div}(\mu \nabla \phi)+\mathrm{q}_{\phi}
$$

where, $\phi$ is velocity component $V_{1}$ and $V_{2} . q_{\phi}$ in this case, represent a gradient of pressure in momentum equation which known as source term.

Finite volume discretisation: According to SIMPLE algorithm the continuity equation is discretised to form pressure correction equation. For unstructured grid the equation is (Mathur and Murthy, 1997):

$\mathrm{a}_{\mathrm{p}} \mathrm{p}^{\prime}=\sum_{\mathrm{nb}} \mathrm{a}_{\mathrm{nb}} \mathrm{p}_{\mathrm{nb}}^{\prime}+\mathrm{b}$

$\mathrm{a}_{\mathrm{nb}}=-\frac{\rho_{\mathrm{f}}\left(\Omega_{\mathrm{p}}+\Omega_{\mathrm{nb}}\right) \overrightarrow{\mathrm{A}} \bullet \overrightarrow{\mathrm{A}}}{\left(\overline{\mathrm{a}}_{\mathrm{p}}+\overline{\mathrm{a}}_{\mathrm{pnb}}\right) \mathrm{ds} \overrightarrow{\mathrm{A}} \bullet \overrightarrow{\mathrm{e}}_{\mathrm{s}}}$

$\mathrm{a}_{\mathrm{P}}=\sum_{\mathrm{nb}} \mathrm{a}_{\mathrm{nb}}$

$\overline{\mathrm{a}}_{\mathrm{p}}$ and $\overline{\mathrm{a}}_{\mathrm{pnb}}$ are point coefficient of dicretised momentum equation (Eq. 13), $\Omega_{\mathrm{p}}$ and $\Omega_{\mathrm{nb}}$ are volume of cell $\mathrm{P}$ and cell neighbor nb. $\overrightarrow{\mathrm{A}}$ and $\overrightarrow{\mathrm{e}}_{\mathrm{s}}$ are face area vector and unit vector from cell $\mathrm{P}$ to cell neighbor $\mathrm{nb}$ respectively.

Finite volume discretisation of general transport equation is done by integrating the equation using Green-Gauss's transformation yielding:

$$
\int_{S}(\rho \phi \vec{V}) \cdot \vec{n} d S=\int_{S}(\mu \nabla \phi) \cdot \vec{n} d S+\int_{\Omega} q_{\phi} d \Omega
$$

where the surface integrals are taken over the boundary of a control region, which for numerical solution will be a grid cell and the source term $q_{\phi}$ is integrated over the volume of this region. $\overrightarrow{\mathrm{n}}$ in Eq. 7 is unit vector normal to surface grid cell. For numerical solution, these equations require discretisation so that they may be applied to the finite volume cells of an unstructured mesh overlaid on the solution domain. The equation, for a finite volume grid cell with a finite number $f$ of identifiable plane faces, may be discretised as:

$\sum_{\mathrm{f}}(\rho \phi \overrightarrow{\mathrm{V}})_{\mathrm{f}} \cdot \overrightarrow{\mathrm{n}} \mathrm{A}_{\mathrm{f}}=\sum_{\mathrm{f}}(\mu \nabla \phi)_{\mathrm{f}} \cdot \overrightarrow{\mathrm{n}} \mathrm{A}_{\mathrm{f}}+\mathrm{q}_{\phi} \Omega$

where the subscript $f$ denotes value of the variables for the face $f . A_{f}$ is the surface area of face $f$, whilst quantities such as $\phi_{\mathrm{f}}$ and $\overrightarrow{\mathrm{V}}_{\mathrm{f}}$ are interpreted as face average values of the scalar $\phi$ and velocity vector $\overrightarrow{\mathrm{V}}$ respectively.

$\phi_{\mathrm{f}}$ can be approximated through reconstruction from upstream cell value as following:

$\phi_{\mathrm{f}}=\phi_{\mathrm{P}}+(\nabla \phi) \bullet \overrightarrow{\mathrm{r}_{\mathrm{Pf}}}$

$\phi_{\mathrm{P}}$ and $\overrightarrow{\mathrm{r}_{\mathrm{Pr}}}$ are $\phi$ at upstream cell centre and distance vector from upstream cell centre to centre of face $f$ respectively as shown in Fig. 1.

The diffusive term is approximated according to Mathur and Murthy (1997) as following:

$$
\begin{aligned}
& (\mu \nabla \phi)_{\mathrm{f}} \overrightarrow{\mathrm{n}} \mathrm{A}_{\mathrm{f}}=\mu_{\mathrm{f}} \frac{\left(\phi_{\mathrm{P}}-\phi_{\mathrm{nb}}\right)}{\mathrm{ds}} \frac{\overrightarrow{\mathrm{A}} \bullet \overrightarrow{\mathrm{A}}}{\overrightarrow{\mathrm{A}} \bullet \overrightarrow{\mathrm{e}}_{\mathrm{s}}}+ \\
& \mu_{\mathrm{f}}\left((\nabla \phi)_{\mathrm{f}} \bullet \overrightarrow{\mathrm{A}}-(\nabla \phi)_{\mathrm{f}} \bullet \overrightarrow{\mathrm{e}_{\mathrm{s}}} \frac{\overrightarrow{\mathrm{A}} \bullet \overrightarrow{\mathrm{A}}}{\overrightarrow{\mathrm{A}} \bullet \overrightarrow{\mathrm{e}_{\mathrm{s}}}}\right)
\end{aligned}
$$

Gradient of field variable on face centre, $(\nabla \phi)_{\mathrm{f}}$, is calculated as average of gradient at cell centre $\mathrm{P}$ and cell neighbor, nb (Fig. 1). The cell centre gradient itself is estimate as:

$$
\nabla \phi=\frac{\mathrm{a}}{\Omega} \sum_{\mathrm{f}} \phi_{\mathrm{f}} \overrightarrow{\mathrm{A}}
$$

a is a limiter used to avoid introducing Local extrema.

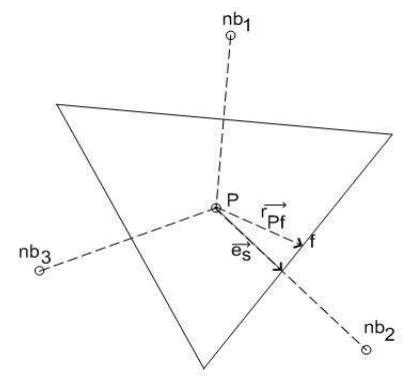

Fig. 1: Finite volume grid 
The last part of discretised equation is a source term which can be estimated as:

$\mathrm{q}_{\phi} \Omega=\sum_{\mathrm{f}} \mathrm{p}_{\mathrm{f}} \overrightarrow{\mathrm{A}} \bullet \overrightarrow{\mathrm{i}}_{\mathrm{j}}$

Where:

$\overrightarrow{\mathrm{i}_{\mathrm{j}}}=$ Unit vector in Cartesian coordinate

$\overrightarrow{i_{1}}=$ Unit vector in $\mathrm{X}$ direction

$\overrightarrow{\mathrm{i}_{2}}=$ Unit vector in Y direction

$P_{f}=$ Pressure at face of control volume which can be estimate using linear interpolation from pressure of cell $\mathrm{P}$ and cell neighbor $\mathrm{nb}$

The face velocity vector $\overrightarrow{V_{f}}$ of convective term (Eq. 8) or in form of mass flux $\dot{\mathrm{m}}_{\mathrm{f}}=(\rho \overrightarrow{\mathrm{V}})_{\mathrm{f}} \cdot \overrightarrow{\mathrm{n}} \mathrm{A}_{\mathrm{f}}$ is calculated using momentum interpolation which will be discussed in the momentum interpolation subsection.

Substitute Eq. 9, 10, 12 and 15 to Eq. 8 and after some mathematical arrangement dicretised momentum equation can be written as following form:

$\overline{\mathrm{a}}_{\mathrm{p}} \phi=\sum_{\mathrm{nb}}^{-} \overline{\mathrm{a}}_{\mathrm{nb}} \phi_{\mathrm{nb}}+\overline{\mathrm{b}}$

\section{Momentum interpolation:}

Current momentum interpolation: It is well known that the combination of a collocated grid arrangement with the use of linear interpolation for estimation of face velocity in continuity equation and estimation of pressure gradient in momentum equation (Eq. 9) cause checkerboard oscillation. Rhie and Chow (1983) first solved this problem for structured collocated grids by modifying the interpolated face velocities by added a diffusive term. Rhie and Chow idea can be written as following:

$$
\mathrm{V}_{\mathrm{f}}=\overline{\mathrm{V}_{\mathrm{f}}}-\frac{\Omega}{\overline{\mathrm{a}}_{\mathrm{p}}}\left(\left(\frac{\partial \mathrm{p}}{\partial \mathrm{n}}\right)_{\mathrm{f}}+\overline{\left(\frac{\partial \mathrm{p}}{\partial \mathrm{n}}\right)_{\mathrm{f}}}\right)
$$

$\Omega$ and $\bar{a}_{\mathrm{p}}$ represent average of cell volume and centre coefficient of discretised momentum equation in left and right of face f. $\bar{V}_{f}$ is average of velocity in left and right face f. Mathur and Murthy (1997) applied the Rhie and Chow (1983) idea to unstructured grid and write the equation in form of mass flux instead of face velocity:

$$
\begin{aligned}
\dot{\mathrm{m}}_{\mathrm{f}}= & \rho \overrightarrow{\mathrm{A}} \bullet\left(\frac{\overrightarrow{\mathrm{V}}_{\mathrm{P}}+\overrightarrow{\mathrm{V}}_{\mathrm{nb}}}{2}\right)-\frac{\rho_{\mathrm{f}}\left(\Delta \Omega_{\mathrm{p}}+\Delta \Omega_{\mathrm{nb}}\right)}{\mathrm{a}_{\mathrm{p}}+\mathrm{a}_{\mathrm{pnb}}} \\
& \left(\frac{\mathrm{P}_{\mathrm{P}}-\mathrm{P}_{\mathrm{nb}}}{\mathrm{ds}}-\nabla \mathrm{p}_{\mathrm{f}} \bullet \overrightarrow{\mathrm{e}}_{\mathrm{s}}\right) \frac{\overrightarrow{\mathrm{A}} \bullet \overrightarrow{\mathrm{A}}}{\mathrm{A} \bullet \overrightarrow{\mathrm{e}}_{\mathrm{s}}}
\end{aligned}
$$

where, ds and $\vec{e}_{\mathrm{s}}$ are distance and unit vector from cell centre $\mathrm{P}$ to centre of neighbor cell. First part of Mathur's equation is linear interpolation estimation of face value velocity, $\overrightarrow{\mathrm{V}}_{\mathrm{f}}$ in Rhie and Chow (1983) equation, Eq. 14. Similar to the first part the second part is linear interpolation estimation of second part of Rhie and Chow's equation. Because of it was based on linear interpolation, to be second order accurate, the face should be in the midway of line from cell centre $\mathrm{P}$ to neighbor cell $\mathrm{nb}$, the face also should be inline with line P-nb or gradient of line P-f and gradient of line $n b-f$ are same. But, It is not a case in most technical problem where unstructured grid is used in order to accommodate a complex geometry. So that accuracy of momentum interpolation will be down to first order.

Improvement of momentum interpolation: Mass flux on face $f$ of unstructured mesh (Fig. 2) can be calculated using velocity on the face as following:

$\dot{\mathrm{m}}_{\mathrm{f}}=\rho \mathrm{A}_{\mathrm{nx}} \mathrm{u}_{\mathrm{f}}+\rho \mathrm{A}_{\mathrm{ny}} \mathrm{v}_{\mathrm{f}}$

where, $A_{n x}, A_{n y}, u_{f}, v_{f}$ are component of face area normal to $\mathrm{x}$ and $\mathrm{y}$ direction and face velocity in $\mathrm{x}$ and $\mathrm{y}$ direction respectively. $\mathrm{u}_{\mathrm{f}}$ and $\mathrm{v}_{\mathrm{f}}$ can be reconstructed form face velocity of imaginary face f', mass flux then can be written as:

$\dot{\mathrm{m}}_{\mathrm{f}}=\rho \mathrm{A}_{\mathrm{nx}}\left(\mathrm{u}_{\mathrm{f}},+\nabla \mathrm{u}_{\mathrm{f}}, \bullet \mathrm{dr}\right)+\rho \mathrm{A}_{\mathrm{ny}}\left(\mathrm{v}_{\mathrm{f}},+\nabla \mathrm{u}_{\mathrm{f}} \cdot \bullet \overrightarrow{\mathrm{r}}\right)$

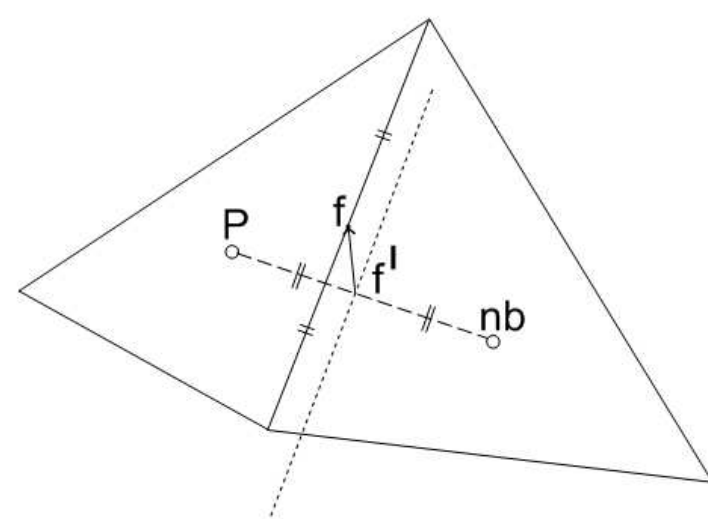

Fig. 2: Unstructured mesh 
Equation 13 is rearranged to be:

$$
\begin{aligned}
\dot{\mathrm{m}}_{\mathrm{f}}= & \left(\rho \mathrm{A}_{\mathrm{nx}} \mathrm{u}_{\mathrm{f}^{\prime}}+\rho \mathrm{A}_{\mathrm{ny}} \mathrm{v}_{\mathrm{f}},\right)+ \\
& \left(\rho \mathrm{A}_{\mathrm{nx}} \mathrm{u}_{\mathrm{f}}, \bullet \overrightarrow{\mathrm{r}}+\rho \mathrm{A}_{\mathrm{ny}} \nabla \mathrm{v}_{\mathrm{f}} \cdot \overrightarrow{\mathrm{dr}}\right)
\end{aligned}
$$

In Eq. $18 \mathrm{dr}$ is a vector from centre of imaginary face f' to centre of face f. $\nabla v_{f}$, and $\nabla u_{f}$, are gradient of velocity on the imaginary face $f$ ' which is calculated as average of left and right cell value. The first term of Eq. 18 is flux on the face f' which can be calculated using Mathur's equation which accurate to second order. The flux then can be written as:

$$
\begin{aligned}
\dot{\mathrm{m}}_{\mathrm{f}}= & \rho \overrightarrow{\mathrm{A}} \bullet\left(\frac{\overrightarrow{\mathrm{V}}_{\mathrm{P}}+\overrightarrow{\mathrm{V}}_{\mathrm{E}}}{2}\right)-\frac{\rho_{\mathrm{f}}\left(\Delta \Omega_{\mathrm{P}}+\Delta \Omega_{\mathrm{E}}\right)}{\mathrm{a}_{\mathrm{p}}+\mathrm{a}_{\mathrm{pnb}}} \\
& \left(\frac{\mathrm{p}_{\mathrm{P}}-\mathrm{p}_{\mathrm{E}}}{\mathrm{ds}}-\nabla \mathrm{p}_{\mathrm{f}} \bullet \overrightarrow{\mathrm{e}}_{\mathrm{s}}\right) \frac{\overrightarrow{\mathrm{A}} \bullet \overrightarrow{\mathrm{A}}}{\overrightarrow{\mathrm{A}} \bullet \overrightarrow{\mathrm{e}}_{\mathrm{s}}}+ \\
& \left(\rho \mathrm{A}_{\mathrm{nx}} \mathrm{u}_{\mathrm{f}} \bullet \overrightarrow{\mathrm{dr}}+\rho \mathrm{A}_{\mathrm{ny}} \nabla \mathrm{v}_{\mathrm{f}} \cdot \overrightarrow{\mathrm{dr}}\right)
\end{aligned}
$$

The first and second term of right hand side of Eq. 19 is a original Mathur's momentum interpolation and the third is a correction term.

\section{Test case:}

Kovasznay flow: In order to test performance of momentum interpolation, 2D Burgers equation in domain $-0.5 \leq \mathrm{x} \leq 1,-0.5 \leq \mathrm{y} \leq 1.5$ was used as test case. Because of it has complexities and non linear effect, it is a good tool to test accuracy of Navier-Stokes solver. The analytical solution of this flow was reported by Kovasznay (1948):

$$
\begin{aligned}
& \mathrm{u}=1-\mathrm{e}^{\mathrm{kx}} \cos 2 \pi \mathrm{y} \\
& \mathrm{v}=\lambda \mathrm{e}^{\mathrm{kx}} \sin 2 \mathrm{y} / 2 \pi \\
& \mathrm{p}=\left(1-\mathrm{e}^{2 \lambda x}\right) / 2 \\
& \lambda=\frac{\operatorname{Re}}{2} \sqrt{\frac{\operatorname{Re}}{4}-4 \pi}
\end{aligned}
$$

Re in this equation is Reynolds number.

The test is carried out on square domain which has two different number of unstructured mesh i.e., 1500 and 1000 as shown in Fig. 3 at $\operatorname{Re}=40$.

Laminar Poisseulli flow: Laminar Poisseulli flow is used to test accuracy of momentum interpolation in distorted mesh (Fig. 5). The domain of $0.5 \mathrm{~m}$ high and 5 $\mathrm{m}$ long have rectangular mesh with distorted mesh in the middle. The analytical solution of the flow is:

$$
\begin{aligned}
& \mathrm{u}=4 \mathrm{~V}_{1 \max }\left(1-\frac{\mathrm{y}}{\mathrm{h}}\right)\left(\frac{\mathrm{y}}{\mathrm{h}}\right) \\
& \mathrm{v}=0 \\
& \mathrm{p}=\mathrm{p}_{0}-8 \mathrm{v} \rho \frac{\mathrm{V}_{1 \max } \mathrm{x}}{\mathrm{h}^{2}}
\end{aligned}
$$

Where:

$\mathrm{p}_{\mathrm{o}} \quad=\mathrm{A}$ constant

$\mathrm{h}=$ High of channel

$\mathrm{V}_{1 \max }=$ Maximal $\mathrm{x}$ velocity which is selected to YIELD Reynolds number of 200 based on high of channel

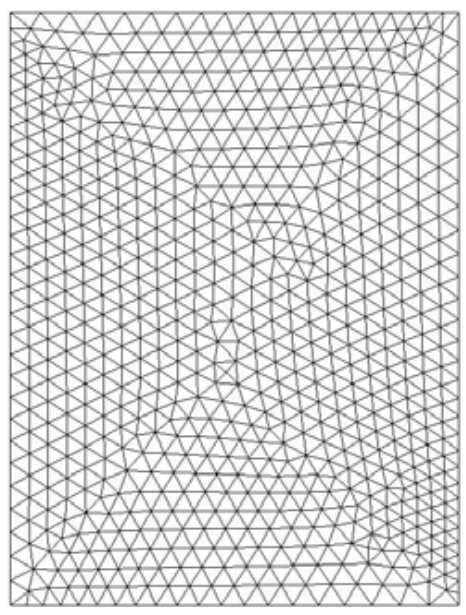

Fig. 3: Geometry and mesh for Kovasznay flow test case

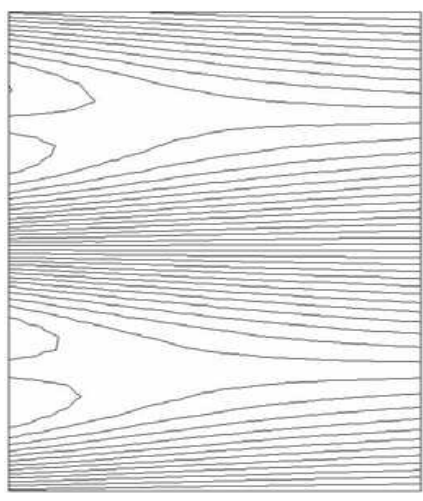

Fig. 4: Streamline of Kovasznay flow

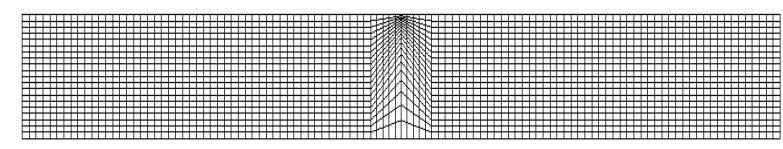

Fig. 5: Geometry and mesh for Poisseulli flow test case 268 
Solving of Kovasznay flow (Fig. 4) and Laminar Poisseulli flow is done by using standard SIMPLE algorithm of Patankar (1980) and Spalding (1980) which also used by Mathur and Murthy (1997). The solution procedure of SIMPLE algorithm is consisting of five steps:

(a) Assume initial value of pressure and velocity at all cell centers

(b) Obtain temporary velocity field by solving momentum equation, Eq. 8

(c) Obtain pressure correction by solving pressure correction equation, Eq. 5

(d) Use pressure correction to update pressure and velocity field using following equation:

$$
\mathrm{p}=\mathrm{p}^{*}+\alpha_{\mathrm{p}} \mathrm{p}^{\prime} \quad \varphi=\varphi^{*}-\frac{\sum_{\mathrm{f}} \mathrm{P}_{\mathrm{f}}^{\prime} \overrightarrow{\mathrm{A}} \overrightarrow{\mathrm{i}_{j}}}{\overline{\mathrm{a}}_{\mathrm{p}}}
$$

where $\mathrm{p}^{*}$ and $\phi^{*}$ are initial value of pressure and temporary velocity field respectively

(e) Repeat steps (b)-(d) where corrected pressure from step (d) is used as new pressure guess until a converged solution is obtained

\section{RESULTS}

Accuracy of standard momentum interpolation and modified momentum interpolation in calculate Kovasznay flow can be compared through the value of $\mathrm{L} 1$ error of $\mathrm{x}$ and $\mathrm{y}$ velocities as shown in Table 1.

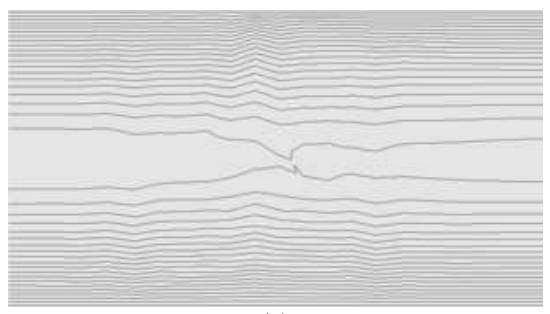

(a)

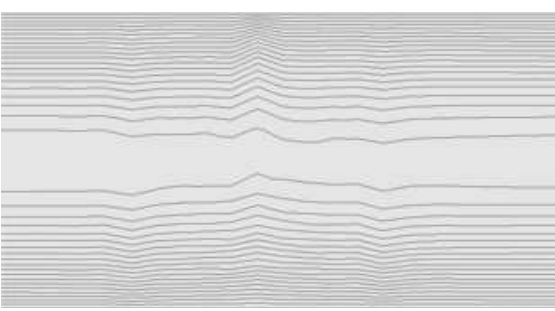

(b)

Fig. 6: X-Velocity contour around distorted mesh. (a) Calculated using standard Mathur's momentum interpolation; (b) Calculated using modification of momentum interpolation
Accuracy of both momentum interpolation in calculate laminar Poisseulli flow is shown through contour of velocity and velocity distribution along centre line of flow channel as shown in Fig. 6 and 7 respectively.

\section{DISCUSSION}

The present research confirms that there are differences of calculation result using standard and modified momentum interpolation.

In Kovasznay's flow solution, face centre of grid used in calculation is lied down almost in the middle between two neighbor cells, so that error of face value of flow variable calculated through linear interpolation as it in Equation 15 is quite low. Both of standard and modified momentum interpolations produce quite similar velocity field and the velocity fields quite close to exact value. The differences of both velocity field, calculated using standard and modified momentum interpolation, is visually indistinguishable. The differences can be shown through L1 error of velocity field. From Table 1 it can be shown that L1 error of velocity field calculated using standard interpolation is almost two fold of it calculated using modified one.

Table 1: Error in Kovasznay flow test $\left(\mathrm{L}_{\mathrm{U}}\right.$ and $\mathrm{L} 1_{\mathrm{V}}$ are $\mathrm{L} 1$ error of $\mathrm{u}$ and $\mathrm{v}$ velocity)

\begin{tabular}{lll}
\hline Mesh size & $\begin{array}{l}\text { Mathur's } \\
\text { momentum intp. }\end{array}$ & $\begin{array}{l}\text { Improved Mathur's } \\
\text { momentum intrp. }\end{array}$ \\
\hline 1500 & L1u $=1.1 \mathrm{e}-2$ & L1u $=6.5 \mathrm{e}-3$ \\
& L1v $=5.8 \mathrm{e}-3$ & L1v $=2.7 \mathrm{e}-3$ \\
1000 & L1u $=2.1 \mathrm{e}-2$ & L1u $=1.4 \mathrm{e}-2$ \\
& L1v $=9.8 \mathrm{e}-3$ & L1v $=5.3 \mathrm{e}-3$ \\
\hline
\end{tabular}

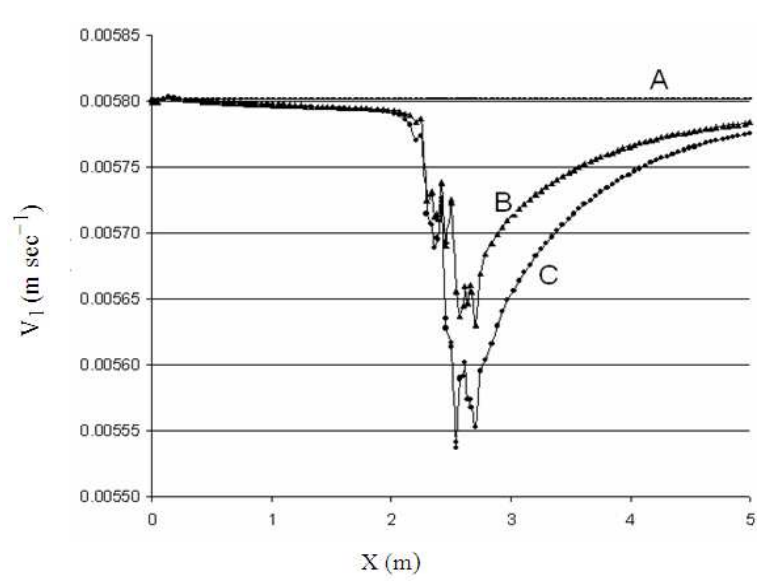

Fig. 7: Velocity along horizontal centre line of channel. (A)Velocity distribution without distortion on the mesh. (B) Velocity distribution calculated using modification of Mathur's momentum interpolation. (C) Velocity distribution calculated using standard Mathur's momentum interpolation 
Laminar Poisseulli flow was calculated using distorted mesh in the middle of domain where face centre almost do not lied down in the middle and inline with both cell neighbor. Difference of two velocity fields, calculated by using standard and modified momentum interpolation, can be shown easily through velocity contour and velocity distribution along horizontal centre-line of flow channel. Velocity contour calculated using standard momentum interpolation is more distorted than velocity contour calculated using modified one, Fig. 6. Whilst error in velocity distribution calculates using standard momentum interpolation is almost double of error in velocity calculated using modified momentum interpolation as shown in Fig. 7.

Some improvements of accuracy have been achieved by adding a correction in order to reduce error caused by using linear interpolation in derivation of momentum-interpolation equation. But linear interpolation is still used to calculate face value of flow variable in equation of gradient of flow variable at cell centre, Eq. 11. Further research to find a correction for this linear interpolation is very interesting in order to reduce error of calculation.

\section{CONCLUSION}

A simple improvement have been done to Mathur's momentum interpolation in order to reduce error of Navier-Stokes Equation solver when face center do not laid down inline and in the middle between two neighbor cell centers. The improvement which done in this work can reduce L1 error of finite volume calculation almost $40 \%$ of its original value as shown in two sample problems used in this study.

\section{REFERENCES}

Choi, S.K., 1999. Note on the use of momentum interpolation method for unsteady flows. Numer. Heat Trans. A., 36: 545-550. DOI: 10.1080/104077899274679

Kovasznay, L.I.G., 1948. Laminar flow behind a twodimensional grid: Proc. the Cambridge Philosop. Soc., $\quad 44: \quad 58-62 . \quad$ DOI: $10.1017 /$ S0305004100023999

Majumdar, S., 1988. Role of under-relaxation in momentum interpolation for calculation of flow with non staggered grids. Numer. Heat Trans., 13: 125-132. DOI: $10.1080 / 10407788808913607$
Mathur, S.R., J.Y. Murthy, 1997. A pressure based methods for unstructured meshes. Numer. Heat Trans. Part B., 31: 195-215. DOI: 10.1080/10407799708915105

Miller, T.F and W. Schmidt, 1988. Use of a pressureweighted interpolation method for the solution of incompressible Navier-stokes equations on a non staggered grids system: Numer. Heat Trans., 14: 213-233. DOI: 10.1080/10407788808913641

Nie, J.H., Z.Y. Li, Q.W. Wang and W.Q. Tao, 2000. A method for viscous incompressible flows with simplified collocated grid system. Proceeding of the 21st Century Symposium on Energy Engineering in the 21st Century, Jan. 9-13, Institute of Thermal Engineering, Hong Kong, pp: 177-183.

Papageorgakopoulos, J., G. Arampatzis, D. Assimacopoulos and N.C. Markatos, 2000. Enhancement of the momentum interpolation method on non-staggered grids. Int. J. Numer. Methods Fluids, 33: 1-2. DOI: 10.1002/(SICI)1097-0363(20000515)33:1<1::AIDFLD978>3.3.CO;2-S

Patankar, S.V., 1980. Numerical Heat Transfer and Fluid Flow. 1st Edn., Taylor and Francis, New York, ISBN 10: 0891165223, pp: 214.

Rhie, C.M. and W.L. Chow, 1983. Numerical study of the turbulent flow past an air foil with trailing edge separation. AIAAJ, 21: 1525-1532. DOI: $10.2514 / 3.8284$

Spalding, D.B., 1980. A mathematical modeling of fluid dynamics, heat transfer and mass transfer processes. Imperial College.

Yu, B. and Y. Kawaguchi, 2002. Checkerboard pressure prediction due to the under relaxation factor and time step size for non staggered grid with momentum interpolation method: Numerical Heat Trans. Part B., 41: 85-94. DOI: 10.1080/104077902753385027

Yu, B., W. Tao, J. Wei, Y. Kawaguchi and T. Tagawa et al., 2002. Discussion on momentum interpolation methods for collocated grids of incompressible flow. Numer. Heat Trans. Part B., 42: 141-166. DOI: 10.1080/10407790190053879 